\title{
Power Aware Routing in Mobile Ad Hoc Networks by using Power Aware Matrices
}

\author{
Navin Mani Upadhyay \\ Assistant Professor \\ Department of CSE \\ Ashoka Institute of Technology \\ \& Management, Varanasi \\ Uttar Pradesh-221007
}

\author{
Kumari Soni \\ Assistant Professor \\ Department of CSE \\ Ashoka Institute of Technology \\ \& Management, Varanasi \\ Uttar Pradesh-221007
}

\author{
Arvind Kumar \\ Assistant Professor \\ Department of CSE \\ Ashoka Institute of Technology \\ \& Management, Varanasi \\ Uttar Pradesh-221007
}

\begin{abstract}
Power consumption is a crucial design concern in Wireless ad hoc networks since wireless nodes are typically battery limited. It might not be possible to replace/recharge a mobile node that is powered by batteries. To take full advantage of life time of nodes, traffic should be routed in a way that power consumption is minimized. Power Aware Routing is a consideration in a way that it minimizes the energy consumption while routing the traffic, aims at minimizing the total power consumption of all the nodes in the network, minimizing the overhead etc. and thus, at maximizing the lifespan of the network using some Power Aware Routing Protocols. They minimize either the active communication energy required to transmit or receive packets or the inactive energy consumed when a mobile node stays idle but listens to the wireless medium for any possible communication requests from other nodes. Transmission power control, load distribution and power management approaches are used to minimize active communication energy.
\end{abstract}

\section{General Terms}

Network Security, Mobile Ad-Hoc Networks, Algorithms.

\section{Keywords}

Power Aware Routing, Transmission Power Control Approach; Load Distribution Approach;

\section{INTRODUCTION}

The main objective of power aware routing protocols is to minimize the power consumption and maximize the network lifetime. The network lifetime is defined up to the moment when a node runs out of its own battery power for the first time. If a node stops its operation, it can result in network partitioning and interrupt communication. The main objective of this paper is to make an explicit literature survey on power aware routing protocols based on transmission power control approach, load distribution approach and sleep/power down approach.

\section{POWER AWARE ROUTING}

Power Aware Model: The mobile nodes in MANET are connected to other mobile nodes. These mobile nodes are free to transmit, i.e. send or receive the data packets to or from other nodes respectively, and require power for such activities. There are 4 important power components [2]: (1) Transmission Power (2) Reception Power (3) Idle Power and (4) Overhearing Power [Fig. 2.1].Transmission PowerWhenever a node sends data packet to other nodes in the network, some amount of energy is required for transmission and such energy is called Transmission Energy $\left(\mathrm{T}_{x}\right)$ of that node and this energy is dependent on size of the data packet. On sending the data packet, some amount of power is consumed. The transmission energy is formulated as:

$$
\begin{gathered}
\mathrm{T}_{x}=\underset{ }{\left(330 * \mathrm{P}_{\text {length }}\right) / 2 * 10^{6}} \\
\text { and } \\
\mathrm{P}_{t}=\mathrm{T}_{x} / \mathrm{T}_{t}
\end{gathered}
$$

Where $\mathrm{T}_{x}$ is transmission energy, $\mathrm{P}_{T}$ is Transmission Power, $\mathrm{T}_{t}$ is the time taken to transmit a data packet and $\mathrm{P}$ length is the length of data packet in bits.

\section{RECEPTION POWER}

Whenever a node receives data packet from other nodes then some amount of energy is taken by the node to receive data packet, which is called Reception Energy $\left(\mathrm{R}_{x}\right)$. On receiving the data packet, some amount of power is consumed. Reception Energy is formulated as:

$$
\mathrm{R}_{x}=(230 * \mathrm{P} \text {-length }) / 2 * 10{ }^{6} \text { and } \mathrm{P}_{R}=\mathrm{R}_{x} / \mathrm{T}_{r},
$$

Where $\mathrm{R}_{x}$ is the Reception Energy, $\mathrm{P}_{R}$ is the Reception Power, $\mathrm{T}_{r}$ is a time taken to receive data packet, and P-length is the length of data packet in bits. Idle Power-In this situation, node neither transmits nor receives any data packets. Power is consumed because it needs to listen to the wireless medium continuously in order to detect a packet that it should receive, so that the node can then switch into receiving mode from idle mode. Idle power is a wasted power that should be eliminated or reduced to a minimum. Thus, Idle Power is:

$$
\mathrm{P}_{I}=\mathrm{P}_{R},
$$

Where $\mathrm{P}_{I}$ is Idle Power and $\mathrm{P}_{R}$ is Reception Power. Overhearing Power- In this case a node picks up the data packets that are destined to other nodes and this is called overhearing and it may consume power. This power is called overhearing power. Unnecessarily receiving such data packets will cause power consumption. Then power consumed in overhearing is:

$$
\mathrm{P}_{\text {over }}=\mathrm{P}_{R},
$$


Where $\mathrm{P}_{\text {over }}$ is Overhearing Power and $\mathrm{P}_{R}$ is Reception Power.

\section{POWER AWARE METRICS}

The main objective of power aware metrics is to carefully share the cost of routing which will ensure that node and network life is increased. These power aware metrics [16] result in power efficient routes, which are detailed below. Minimize Energy consumed per packet: This is one of the most obvious metrics that conserves power efficiently.

Assume that some packet $\mathrm{j}$ traverses $n_{1}, \ldots . ., n_{k}$ nodes where

$n_{1}$ is the source and $n_{k}$ the destination. Let $\mathrm{T}(\mathrm{a}, \mathrm{b})$ denote the energy consumed in transmitting and receiving one packet over one hop from a to $b$. Then the energy consumed for packet $\mathrm{j}$ is,

$$
e_{j}=\sum_{i=1}^{k-1} T\left(n_{i}, n_{i+1}\right)
$$

Thus, the goal of this metric is to $\operatorname{minimize} e_{j}$, for all $\mathrm{j}$ [Fig.2]. It is easy to see that this metric will minimize the average energy consumed per packet. In fact it is interesting to observe that, under light loads, the routes selected using this metric will be identical to routes selected by shortest-hop routing. This is not a surprising observation because, if we assume that $\mathrm{T}(\mathrm{a}, \mathrm{b})=\mathrm{T}=$ Constant, for all $(\mathrm{a}, \mathrm{b}) \in \mathrm{E}$, where $\mathrm{E}$ is the set of all edges, then the power consumed is $(\mathrm{k}-1) \mathrm{T}$. To minimize this value, we simply need to minimize $\mathrm{k}$ which is equivalent to finding the shortest-hop path. In some cases, however, the route selected using this metric may differ from the route selected by shortest-hop routing. Thus, if one or more nodes on the shortest-hop path are heavily loaded, the amount of energy spent in transmitting one packet over one hop will not be a constant since we may spend variable amount of energy (per hop) on contention. Thus, this metric will tend to route packets around congested areas (possibly increasing hop-count). One serious drawback of this metric is that nodes will tend to have widely differing energy consumption profiles resulting in early death of some of the nodes. Consider the network illustrated in Figure 3.Here, node 6 will be selected as the route for packets going from 0-3, 1-4 and 2-5. As a result, node 6 will spend its battery resources at a faster rate than the other nodes in the network and will be the first to die.

Maximum Time to Network Partition: One of the difficulties in implementing this metric is that given a network topology, using the max-flow-min-cut theorem, we can find a minimal set of nodes (the cut-set) the removal of which will cause the network to partition. The routes between these two partitions must go through one of these critical nodes.

A routing procedure therefore must divide the work among these nodes to maximize the life of the network. If we don't ensure that these nodes use up their power at equal rates, then we will observe that delays will increase as soon as one of these nodes dies. Problem is similar to the load balancing problem where tasks need to be sent to one of the many servers available so that the response time is minimized. This is known to be a NP-complete problem. Since nodes in different partitions independently take routing decisions, we cannot achieve the global balance required to maximize the network partition time while minimizing the average delay [25]. As the power consumption is dependent on the length of the packet, we cannot decide optimal routes without the knowledge of future packet arrivals. If all the packets are of same length, then we can ensure equal power drain rate among these nodes by selecting these nodes in a round-robin fashion in routing. Minimum Variance in node power levels: This metric ensures that all the nodes in the network remain up and running together for as long as possible. This problem is similar to load sharing in distributed systems where the objective is to minimize response time while keeping the amount of unfinished work in all nodes the same. This is an intractable problem, because the execution times of future arrivals are not known. Join the Shortest Queue (JSQ) policy can be used to achieve this goal. Here each node sends traffic through a neighbor with the least amount of data waiting to be transmitted. If all packets are of same length, then we can achieve the equal power drain rate by choosing next hop in a round-robin fashion so that on the average, all nodes process equal number of packets.

\section{MINIMIZE COST PER PACKET}

This metric is used to maximize the life of all nodes in the network. The path selected using this metric should be such that nodes with depleted power reserves do not lie on many paths [14]. Let $f_{i}\left(x_{i}\right)$ be a function that denotes the node cost or weight of node I, where $x_{i}$ represents the total energy spent by node $i$. The total cost of sending a packet along some path is the sum of costs at individual nodes from $n_{1}$ to $n_{k}$ via intermediate nodes, $n_{2} n 3 n 4, \ldots, n_{k-1}$ and can be represented as

$$
c_{j}=\sum_{i=1}^{k-1} f_{i}\left(x_{i}\right)
$$

The goal of this metric is to Minimize $c_{j}$, for all packets $\mathrm{j}$. If $f_{i}$ is a monotonically increasing function, then nodes will not be overused thus increasing their life, where $f_{i}$ can be tailored to reflect a battery's remaining life time.

$$
f_{i}\left(x_{i}\right)=\left(1 /\left(1-g\left(x_{i}\right)\right)\right.
$$

where $g\left(x_{i}\right)$ is the normalized battery capacity.

\section{PAAODV PROTOCOL}

Power Aware AODV (PAAODV) protocol for ad hoc networks is an enhancement of existing AODV ad hoc routing protocol. The main objective of PAAODV is to optimally reduce power consumption to a minimum power level in MANET without disruption of network connectivity. As a result of it, the overall power consumed in transmission of overhead packets is significantly reduced. The control messages used in AODV protocol is modified. PAAODV [21] works in two phases that is route-discovery and link-by-link power adjustment $[8-9,13]$. In the route discovery process, different power levels are used to determine a route consuming minimum power for transmission of packets. In this case, a source node attempts first to discover a path with a low power level. Transmit power levels of nodes in an ad hoc network using PAAODV is controlled to minimum levels. Controlling the transmit power level is performed due to two reasons: (i) transmit power level is directly related to the available power at the node and (ii) network connectivity is significantly affected by transmit power. The basic principle of PAAODV is that nodes in the network should control the transmit power in order to maintain the network connectivity. As shown in Fig. 3.4, node p1 is transmitting a packet to node 
p2 and node p3 is transmitting a packet to node p4. In Fig.4(a), both transmissions are successful since they do not interfere with each other. In Fig.4(b), communications interfere with each other due to high transmit power level and hence, cannot be successful. In Fig.4(c), as the transmit power is too low, the network connectivity fails and the communications fail too. Thus, for the communication to take place, the power level should be as low as possible, but at the same time, the connectivity should be maintained. This transmit power, regarded as minimum transmit power level, i.e. $P_{\min }$, can be calculated

$$
P_{\min }(d)=p_{t h} d^{\gamma} / K
$$

Where $d$ is the distance between two nodes, $\gamma$ is the path loss component and ' $K$ ' is constant. Authors have assumed $\gamma=4$ during implementation, which is the path loss component for a two-ray ground model. The value of $P_{t h}$ for IEEE based network is $3.653 \times 10^{10} \mathrm{~mW}$. So the minimum power consumption is calculated as:

$$
\mathrm{E}_{\text {min }}=K_{3} D d^{4}+K_{2}
$$

Where $\mathrm{K}_{3}=2.8 \times 10^{-10} \mu \mathrm{J} / \mathrm{byte}$. The equation reveals that $\mathrm{E}_{\text {min }}$ depends upon $\mathrm{d}$, the distance between two nodes. Otherwise for a fixed transmits power level, the power consumption can be calculated as:

$$
\mathrm{E}_{\text {max }}=K_{4} \mathrm{D}+\mathrm{K}_{2}
$$

Where $\mathrm{K}_{4}=1.62 \mu \mathrm{J} / \mathrm{byte}$. Thus the amount of power saved can be obtained as:

$$
\mathrm{S}(\mathrm{D}, \mathrm{d})=\mathrm{E}_{\text {max }}-\mathrm{E}_{\text {min }}
$$

\section{LOAD DISTRIBUTION APPROACH}

The specific objective of load distribution approach[10] is to balance the energy usage of all mobile nodes by selecting a route with underutilized nodes rather than the shortest route. This may result in longer routes, but packets are routed only through energy rich intermediate nodes. Protocols based on this approach do not necessarily provide the lowest energy route,but prevent certain nodes from being overloaded and thus,ensures longer network lifetime. This subsection discusses two such protocols:Localized Energy-Aware Routing(LEAR) and Conditional Max-Min Battery Capacity Routing protocols. Fig.5 shows the detailed study about Power controller graph of initial 10 packets, Power graph for Packet arrivals, Packet flow graph and Aggregate Throughput.

\section{CONCLUSION}

Energy efficiency is one of the main problems in a MANET, especially in designing a routing protocol. In this paper, we performed an exclusive survey and classified a number of power-aware routing schemes. In many cases, it is difficult to compare them directly since each method has a different goal with different assumptions and employs different means to achieve the goal. When the transmission power is controllable, the optimal adjustment of the power level is essential not only for energy conservation but also for the interference control. When node density or traffic density is far from uniform, a load distribution approach must be employed to alleviate the energy imbalance problem. Therefore, more research is needed to combine and integrate some of the protocols presented in this paper to keep MANETs functioning for a longer duration.

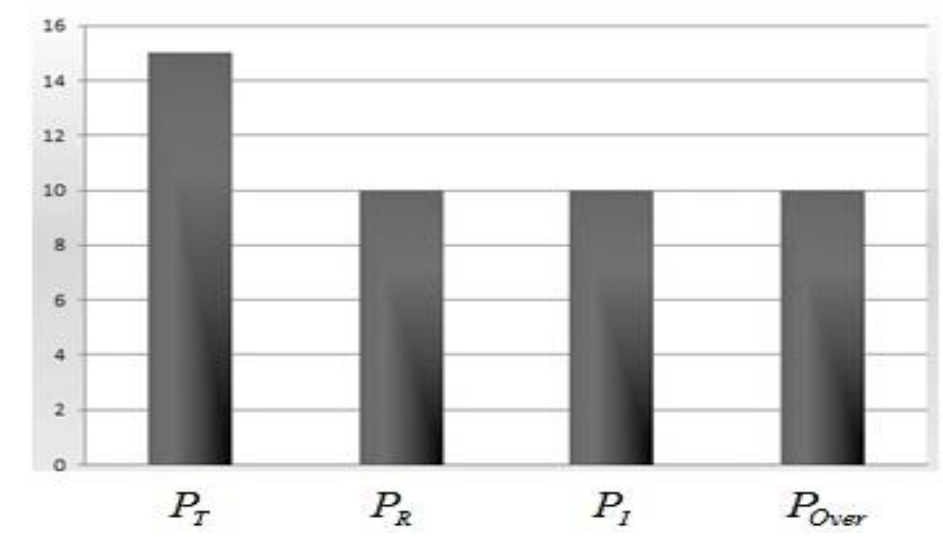

Fig 1: Power Consumption Model of Ad Hoc Nodes

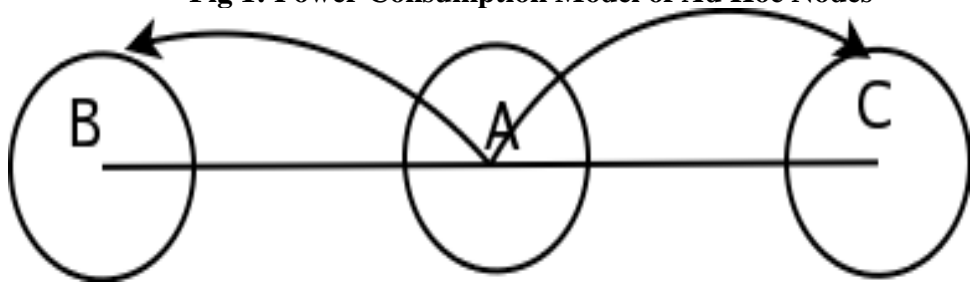

Fig 2: Unnecessary power consumption 


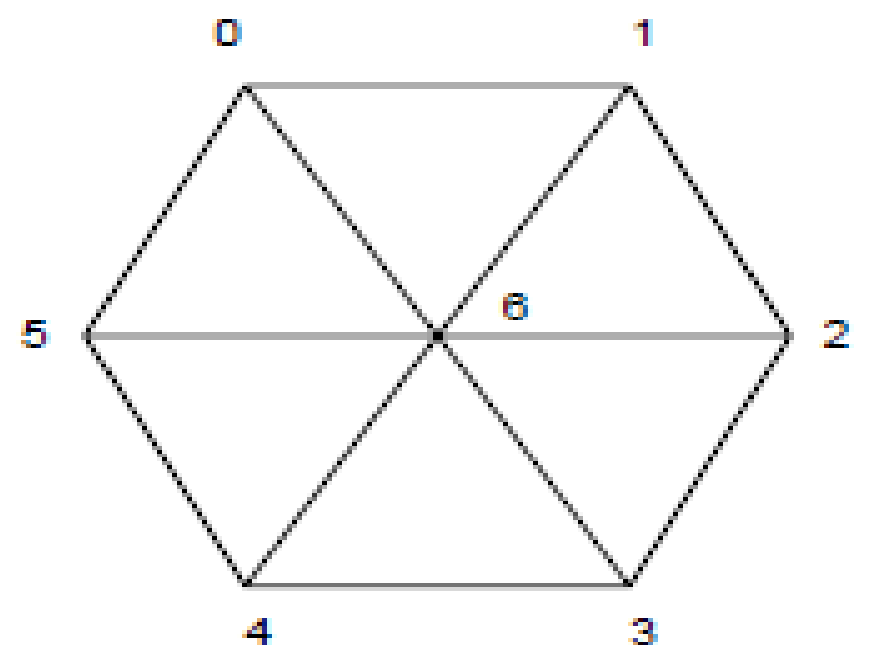

Fig 3: Energy Packet as a metric

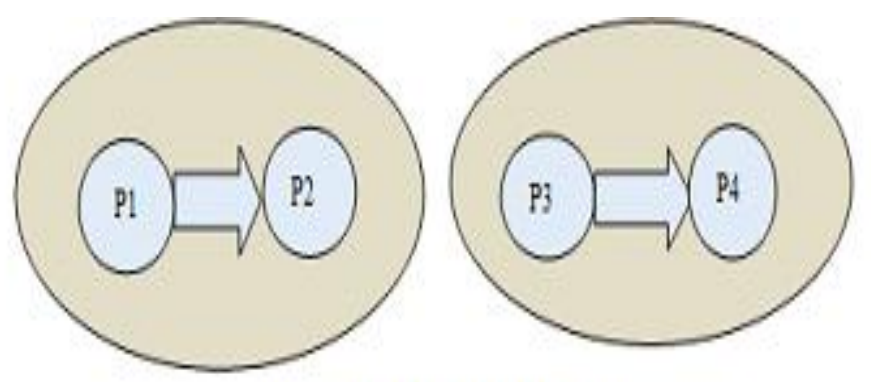

(a) Minimum transmit powrer

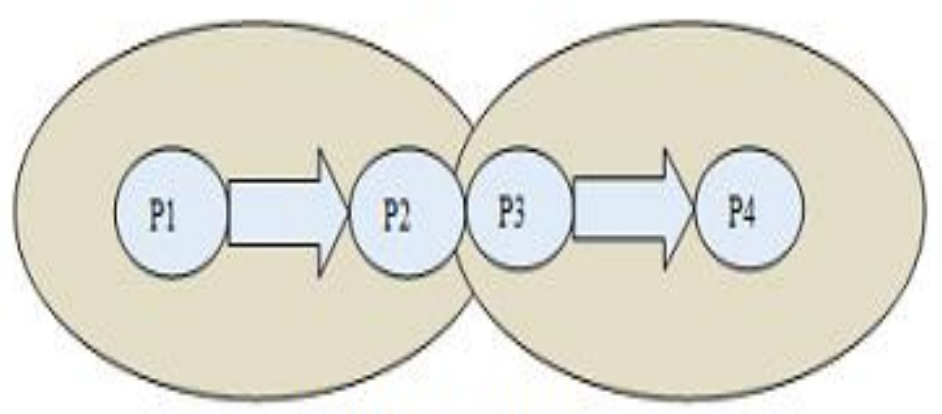

(b) High transmit power
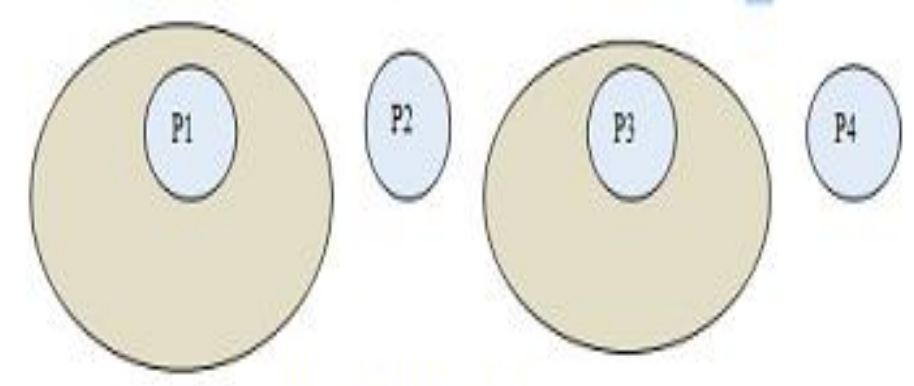

(c) Low trans mit porrer

Fig 4: Effect of transmit power control on network connectivity 


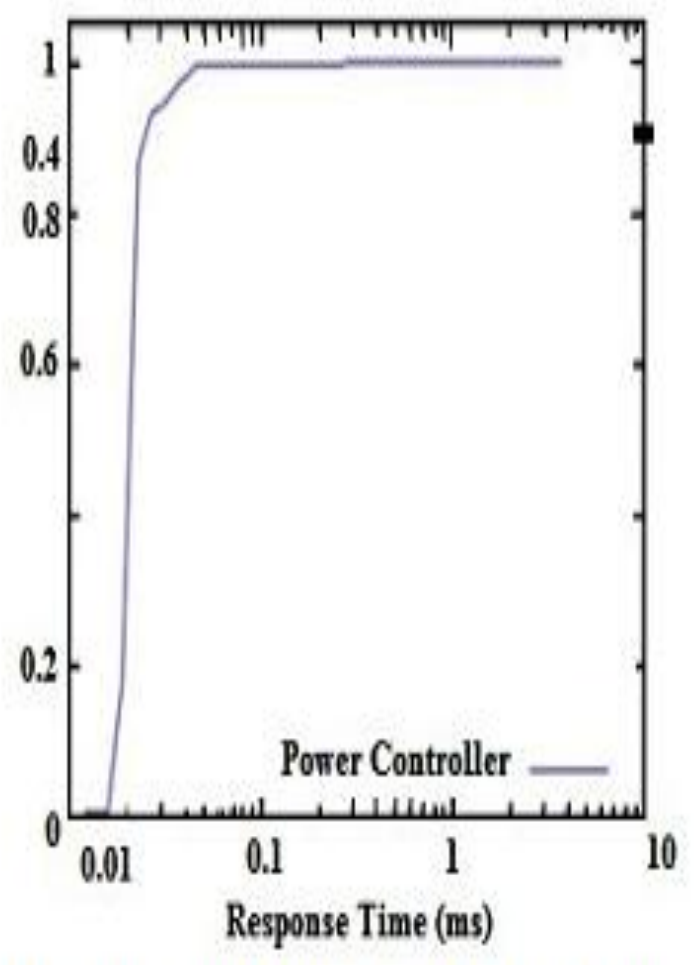

(a) Power Controller Graph for initial 10 Packets

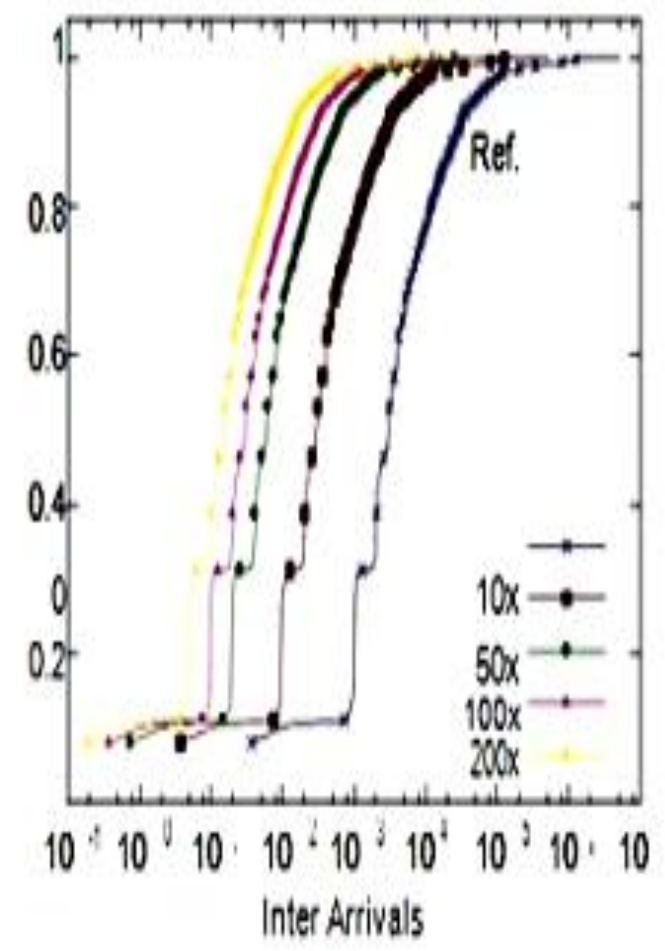

(b) Power Graph for Packets Arrivals
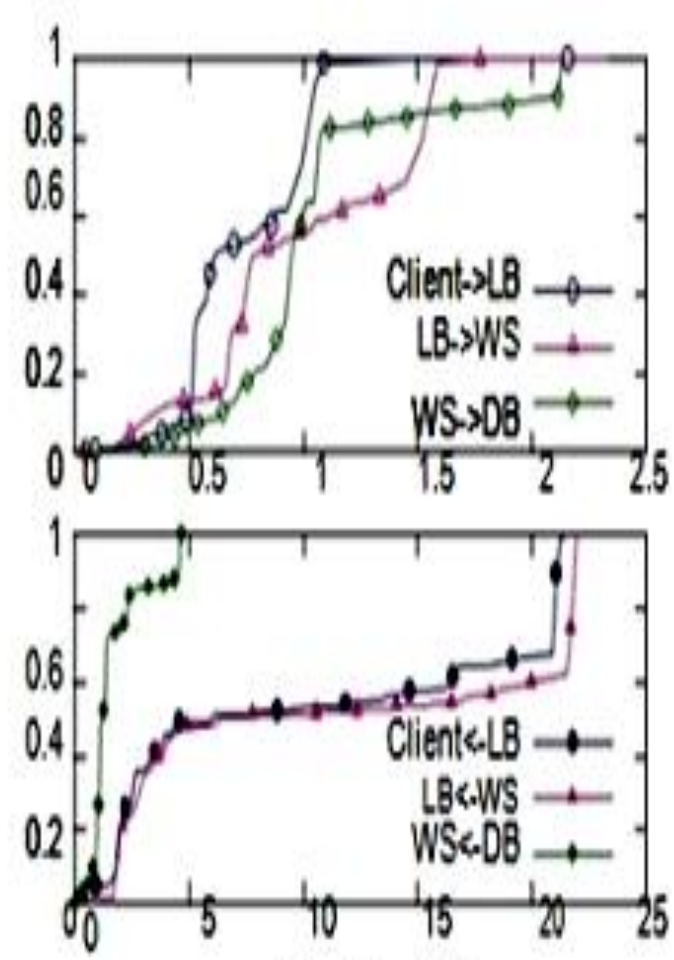

Flow Sizes [KB]

(c) Packets Flow Graph in KB

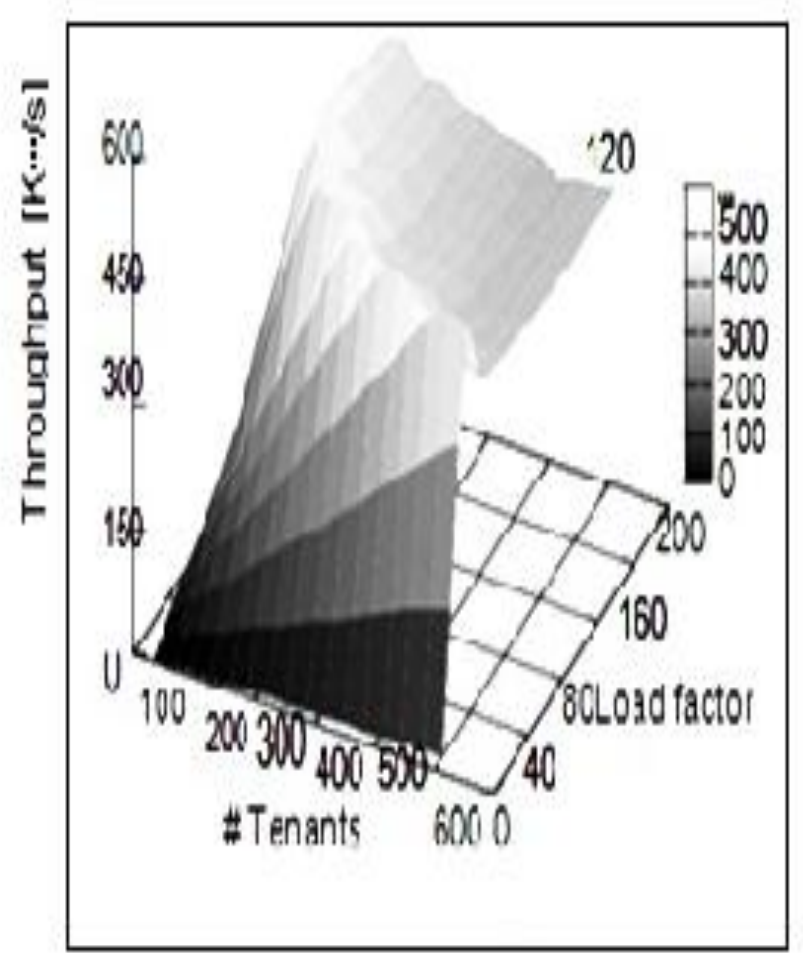

(d) Aggregate throughput of 600 Packets

Fig 5: Effect of power control for flow of packets and corresponding throughput on network connectivity 


\section{REFERENCES}

[1] Kush Ashwani, Gupta Phalguni, Kumar Ram 2005 Performance Comparison of Wireless Routing Protocols. Journal of the CSI, 35(2).

[2] Taneja Sunil, Kush Ashwani, 2010 A Survey of Routing Protocols in Mobile Ad Hoc Networks. International Journal of Innovation, Management and Technology, 1(3).

[3] Pathak, P. H.; Dutta, R. 2011 A Survey of Network Design Problems and Joint Design Approaches in Wireless Mesh Networks. IEEE Communications Surveys \&Tutorials13(3), 396-428.

[4] Zhang H., Arora A., Choi Y., Gouda M. G. 2005 Reliable Bursty Converge cast in Wireless Sensor Networks. Proceedings of the $6^{\text {th }}$ ACM International Symposium on Mobile Ad HocNetworking andComputing (MobiHoc'05), UrbanaChampaign, IL.

[5] McCabe A., Fredin M., Cullen A. and Axelsson L. 2005 A power consumption study of DSR and OLSR. IEEE Military Communications Conference (MILCOM'05), 1954-1960.

[6] Raju S.R. and Mungara J., 2010, Performance Evaluation of ZRP over AODV and DSR in Mobile Adhoc Networks using Qualnet. European Journal of Scientific Research, 45, 658-674.

[7] Bheemalingaiah M., Naidu M.M., Rao D.S., 2009, Energy aware Clustered based Multipath Routing in Mobile Ad Hoc Networks, I.J. Communications, Network and System Sciences, 2, 91-168.

[8] Almobaideen W., Hushaidan K., Sleit A., Qatawneh M., 2011, A Cluster based Approach for Supporting QoS in Mobile Ad Hoc Networks. International Journal of Digital Content Technology and its Applications, 5(1).

[9] Chen Lei and Heinzelman W. B., 2007, A Survey of Routing Protocols that Support QoS in Mobile Ad Hoc Networks. IEEE Comm. Surveys and Tutorials, 21(6), 30-38.

[10] Chen L. and Heinzelman W.B., 2005, QoS-aware Routing Based on Bandwidth Estimation for Mobile Ad Hoc Networks. IEEE JSAC, Special Issue on Wireless Ad Hoc Networks, 23(3), 561-72.
[11] Mieghem P. Van, Kuipers F.A., 2004, Concepts of exact quality of service algorithms. IEEE/ACM Transaction on Networking 12 (5), 851-864.

[12] Reddy T. B. et al., 2006, Quality of Service Provisioning in Ad Hoc Wireless Networks: A Survey of Issues and Solutions. Journal of Ad Hoc Networks, 4(1),83-124.

[13] Masip-Bruin X., Yannuzzi M., 2006, Research challenges in QoS routing. Computer Communications, $29,563-581$.

[14] Pattanayak B. K., Jagadev A. K., Mishra M. K., Nayak M. R., 2009, A Distributed Cluster Scheme For Bandwidth Management In Multi-hop MANETs. IJCSNS International Journal of Computer Science and Network Security. 9(10).

[15] Rishiwal Vinay, Verma S. and Bajpai S. K., 2009, QoS Based Power Aware Routing in MANETs. International Journal of Computer Theory and Engineering, 1(1), 1793-8201

[16] Deepalakshmi P., Radhakrishnan S., 2009 Ant Colony Based QoS Routing Algorithm For Mobile Ad Hoc Networks," International Journal of Recent Trends in Engineering. 1(1).

[17] Allard, G., Minet, P., Nguyen, D. Q. and Shresta N., 2006, Evaluation of the energy consumption in MANET. Adhoc-Now, Ottawa, Canada.

[18] Upadhyay N. M., Singh Vandana, Kumar Arvind, 2013 , Power Consumption Routing Mechanism in Mobile AdHoc Network: A Survey. Proce. Of NCRTCGS.

[19] Upadhyay N. M., Gaurav Kunal and Kumar Arun, 2014, Modified DSR, an Energy Conserving approach to DSR protocol in MANET. IEEE International conference on Communication and Signal Processing.

[20] Patil Manesh P., 2013, A New Approach of Secure power Aware Routing for Mobile Ad-Hoc Network. International Journal of Computer Applications, 67(3).

[21] Bonetto E., Chiaraviglio Luca, Cuda Davide, Idzikowsk Filip, and Neri Fabio, 2013, Exploiting Traffic Dynamics in Power-Aware Logical Topology Design. Optical communications. 